\title{
Prospect and potential of Burkholderia sp. against Phytophthora capsici Leonian: A causative agent for foot rot disease of black pepper
}

\begin{abstract}
Foot rot disease is a very destructive disease in black pepper in Malaysia. It is caused by Phytophthora capsici Leonian, which is a soilborne pathogenic protist (phylum, Oomycota) that infects aerial and subterranean structures of many host plants. This pathogen is a polycyclic, such that multiple cycles of infection and inoculum production occur in a single growing season. It is more prevalent in the tropics because of the favourable environmental conditions. The utilization of plant growth-promoting rhizobacteria (PGPR) as a biological control agent has been successfully implemented in controlling many plant pathogens. Many studies on the exploration of beneficial organisms have been carried out such as Pseudomonas fluorescens, which is one of the best examples used for the control of Fusarium wilt in tomato. Similarly, P. fluorescens is found to be an effective biocontrol agent against the foot rot disease in black pepper. Nowadays there is tremendous novel increase in the species of Burkholderia with either mutualistic or antagonistic interactions in the environment. Burkholderia sp. is an indigenous PGPR capable of producing a large number of commercially important hydrolytic enzymes and bioactive substances that promote plant growth and health; are eco-friendly, biodegradable and specific in their actions; and have a broad spectrum of antimicrobial activity in keeping down the population of phytopathogens, thus playing a great role in promoting sustainable agriculture today. Hence, in this book chapter, the potential applications of Burkholderia sp. to control foot rot disease of black pepper in Malaysia, their control mechanisms, plant growth promotion, commercial potentials and the future prospects as indigenous PGPR were discussed in relation to sustainable agriculture.
\end{abstract}

Keyword: Phytophthora capsici Leonian; Foot rot disease; Black pepper; Plant growthpromoting rhizobacteria (PGPR) 